\title{
Psychiatric Findings in Suspected and Confirmed Middle East Respiratory Syndrome Patients Quarantined in Hospital: A Retrospective Chart Analysis
}

\author{
Hyun-Chung Kim¹, So-Young Yoo', Bun-Hee Lee ${ }^{2}$, So Hee Lee ${ }^{\circledR}$, and Hyoung-Shik Shin ${ }^{3}$ \\ 'Department of Psychiatry, National Medical Center, Seoul, Republic of Korea \\ ${ }^{2}$ Maum \& Maum Psychiatric Clinic, Seoul, Republic of Korea \\ ${ }^{3}$ Center for Infectious Disease, National Medical Center, Seoul, Republic of Korea
}

Objective Little is known about the psychiatric complications or risk factors for depression in suspected or confirmed Middle East Respiratory Syndrome (MERS) patients quarantined in hospital.

Methods A retrospective chart review was performed of all the patients admitted to the acute MERS inpatient unit at the NMC during the 2015 outbreak.

Results 30 (75\%) were confirmed to be MERS-CoV positive among 40 admitted cases. Among the 24 MERS survivors, 17 (70.8\%) exhibited psychiatric symptoms and $10(41.7 \%)$ received a psychiatric diagnosis and medication during their hospital stay. Suspected MERS patients did not exhibit psychiatric symptoms or receive a psychiatric diagnosis. 27 suspected or confirmed MERS patients (age 41.15 \pm 18.64 , male 37.0\%) completed psychological assessments. A multiple linear regression analysis revealed that the Korean National Health and Nutrition Examination Survey-Short form and the Impact of Event Scale-Revised scores were significantly positively correlated with Patient Health Questionnaire-9 scores.

Conclusion Our findings indicate that the acute treatment of MERS-CoV infections in quarantine had a significant impact on the patients' mental health. Furthermore, assessment of the risk factors for depression may identify vulnerable patients who require psychiatric care and attention during hospital quarantine.

Psychiatry Investig 2018;15(4):355-360

Key Words Middle east respiratory syndrome, Quarantined hospitalization, Psychiatric symptoms, Depression, Emerging infectious diseases.

\section{INTRODUCTION}

A large outbreak of Middle East respiratory syndrome coronavirus (MERS-CoV) infection occurred in South Korea between May and July 2015. The first patient, a so-called super spreader, had the highest transmission rate of the virus, which eventually resulted in 186 confirmed cases. ${ }^{1}$ An increase in MERS-CoV disease activity raised concerns of a pandemic.

\footnotetext{
Received: July 4, 2017 Revised: August 24, 2017

Accepted: October 25, 2017

$\triangle$ Correspondence: So Hee Lee, MD, PhD

Department of Psychiatry, National Medical Center, 245 Eulji-ro, Jung-gu, Seoul 04564, Republic of Korea

Tel: +82-2-2260-7311, Fax: +82-2-2268-5028, E-mail: psyhee@hanmail.net

$\triangle$ Correspondence: Hyoung-Shik Shin, MD, PhD

Center for Infectious Disease, National Medical Center, 245 Eulji-ro, Jung-gu, Seoul 04564, Republic of Korea

Tel: +82-2-2260-7311, Fax: +82-2-2268-5028, E-mail: hyoungsshin@gmail.com

(a) This is an Open Access article distributed under the terms of the Creative Commons Attribution Non-Commercial License (http://creativecommons.org/licenses/bync/4.0) which permits unrestricted non-commercial use, distribution, and reproduction in any medium, provided the original work is properly cited.
}

Middle East respiratory syndrome (MERS) is a lower respiratory tract infection that causes fever, coughing, breathing difficulties, and pneumonia. The condition may progress to acute respiratory distress syndrome and multiorgan failure and leads to death in $20-40 \%$ of those infected. Human-to-human transmission occurs through droplet spread. Owing to the highly contagious nature of MERS, individuals suspected of exposure to the virus are quarantined.

Data from severe acute respiratory syndrome (SARS) outbreaks showed that quarantined patients developed psychiatric symptoms that required psychiatric management. ${ }^{2}$ However, little is known about the psychiatric complications or risk factors for depression in suspected or confirmed MERS patients quarantined in hospital.

The National Medical Center (NMC) considered the MERS outbreak to be a national disaster and served as a nationally designated MERS treatment center. Thus, we retrospectively evaluated our consultation-liaison strategies and psychiatric 
complications for inpatients with MERS. Additionally, we reviewed patient psychological assessments to identify risk factors associated with depression severity.

\section{METHODS}

\section{Study design and participants}

The study was approved by the Institutional Review Board for Human Subjects at the NMC in Seoul, Korea (H-1508057-004). We reviewed the psychiatric charts for all consecutive admissions for suspected or confirmed MERS to the acute MERS inpatient unit at the NMC, from May to July 2015 to obtain information concerning demographic characteristics, previous medical conditions, previous or present psychiatric disorders or psychiatric symptoms, and the results of psychological assessments. Furthermore, descriptions of the experiences of patients with suspected and confirmed MERS were collected through retrospective chart review.

\section{Consultation strategy}

\section{Service delivery}

We as psychiatrists thought it important to make good rapport with the patient from the beginning, as soon as they entered the MERS ward for the first time. To achieve this goal each psychiatrist was assigned to a patient from admission and each psychiatrist, wearing the proper protective equipment, introduced ourselves to the assigned patient face-to-face and informed the patient of daily telephone interviews and symptom screening. This helped to build acquaintance with the patient and give personal familiarity when receiving their first telephone call. Daily telephone calls were offered as psychiatric rounding to check any sleep or mood disturbances. Further face-to-face interview was done if requested by the patient or when acute psychiatric symptoms emerged. These two modes of mental health services were offered to all of the MERS inpatients from day 1 in order to give support and to detect and treat possible psychiatric symptoms.

\section{Protocols}

There were two protocols. One protocol was used for the daily psychiatric telephone rounding and one was a package of checklists for the patients. The checklist package was given to the patient after medical examinations and history taking was done.

A psychologist called all of the patients to ask the questions on the checklist. This allowed collecting and assessing the checklist without direct contact with the patient or the contaminated checklist package.

\section{Psychological measures}

The patients completed the Patient Health Questionnaire-9 (PHQ-9), the Impact of Event Scale-Revised (IES-R), the Korean National Health and Nutrition Examination Survey (KNHANES)-short form, and the Peritraumatic DissociationPosttraumatic Negative Beliefs-Posttraumatic Social Support (PTD-PTNB-PTSS) scale upon admission to the quarantine ward. A psychologist collected data on the second hospital day using a smartphone. Patients with poor physical or mental status were excluded from the psychological assessment using the structured instruments.

The PHQ- 9 is used to monitor the severity of depression. The questionnaire consists of nine items based on the Diagnostic and Statistical Manual of Mental Disorders, fourth edition (DSM-IV) criteria for depression, each scored from 0 to 3 providing a severity score ranging from 0 to 27 . Accordingly, depression severity was defined as: no depression (1-4), mild depression (5-9), moderate depression (10-14), moderately severe depression (15-19), and severe depression (20-27). The Korean version of the PHQ-9 has been shown to be a reliable and valid tool for the screening and assessment of depressed patients (Cronbach's alpha coefficient $=0.81){ }^{3}$

The IES-R was designed to assess symptoms associated with post-traumatic stress disorder (PTSD) following a traumatic life event (MERS infection in our study). Symptoms are measured on 22 self-reported items for a total subjective stress assessment that can be further divided into intrusion, avoidance, and hyperarousal subscales. Individual items describe difficulties associated with stressful events, and participants were asked to indicate the extent to which each item distressed or bothered them over the last 7 days using a rating scale of $0-4$. The Korean IES-R has shown good reliability (Cronbach alpha coefficient $=0.93$ ), test-retest reliability (Cronbach alpha coefficient $=0.91$ ), and validity for the assessment of PTSD symptom severity. ${ }^{4}$

The KNHANES-short form is a shortened version of the KNHANES developed by the Korea Centers for Disease Control and Prevention to assess current stress perception. Most measures of distress are derived from pre-existing structured tools that capture the amount of stress or change associated with stressful events that have occurred over the past month. The KNHANES has been shown to be reliable and valid, suggesting that it may be a useful instrument for assessing current feelings of stress such as burn-out, depression, and anger. ${ }^{5}$ The KNHANES-short form is composed of nine items and responses are rated using a five-point Likert scale.

The PTD-PTNB-PTSS is a shortened version of the Posttrauma Risk Checklist (PRC), which is a validated Korean instrument used to identify the risk factors for PTSD. ${ }^{6}$ The PRC consists of 57 items that measure PTD, PTNB, and PTSS. The 
PTD-PTNB-PTSS scale comprises 18 items selected from the PRC (3 PTD, 10 PTNB, and 5 PTSS items) that have been converted from yes/no answers to a five-point Likert scale.

\section{Statistical analysis}

Patients were divided into two groups based on PHQ-9 scores: those experiencing mild-to-severe depression and those without depression. An independent-sample t-test was used to test for differences in continuous variables between quarantined patients with mild-to-severe depression and those without depression. Pearson's correlation analysis was used to examine the correlations among psychological measures. A multiple linear regression analysis was performed to identify the risk factors for depression in patients with suspected or confirmed MERS. The null hypothesis was rejected at $\mathrm{p}<0.05$. All statistical tests were conducted using the Statistical Package for the Social Science (SPSS) for Windows (ver. 18.0; SPSS Inc., Chicago, IL, USA), and the threshold for twosided significance tests was $\mathrm{p}<0.05$.

\section{RESULTS}

A total of 40 confirmed and suspected MERS cases were admitted to a quarantine ward at the NMC. Of those, 30 (75\%) were confirmed to be MERS-CoV positive. Among the con- firmed MERS patients, 24 survived and 6 died. Of the 34 surviving confirmed or suspected MERS patients, 12 (35.3\%) were male and $22(64.7 \%)$ were female and had a mean age of $46.15 \pm 20.41$ (range, $21-86$ ) years. In total, 20 (58.8\%) of the surviving confirmed and suspected MERS patients reported previous medical conditions and three $(8.8 \%)$ reported previous psychiatric disorders.

Among the confirmed MERS patients, 17 (70.8\%) exhibited psychiatric symptoms and $10(41.7 \%)$ received a psychiatric diagnosis and were prescribed medication during their hospital stay (Figure 1). Suspected MERS patients did not exhibit psychiatric symptoms or receive a psychiatric diagnosis. Of the 17 patients with psychiatric symptoms, 14 complained of experiencing: insomnia $(n=7)$, depressive $\operatorname{mood}(n=5)$, and tension $(n=9)$, and three were disoriented $(n=2)$, had impaired memory $(n=2)$, auditory hallucinations $(n=2)$, and aggressive outbursts $(n=2)$.

\section{Diagnosis}

DSM-IV-text revision criteria were used to diagnose three patients with adjustment disorders, two with depressive disorders, and two with acute stress disorders after they recovered from delirium. Two patients were diagnosed with anxiety disorders, one of whom had comorbid mild neurocognitive disorder. The cognitive function of a patient previously diagnosed

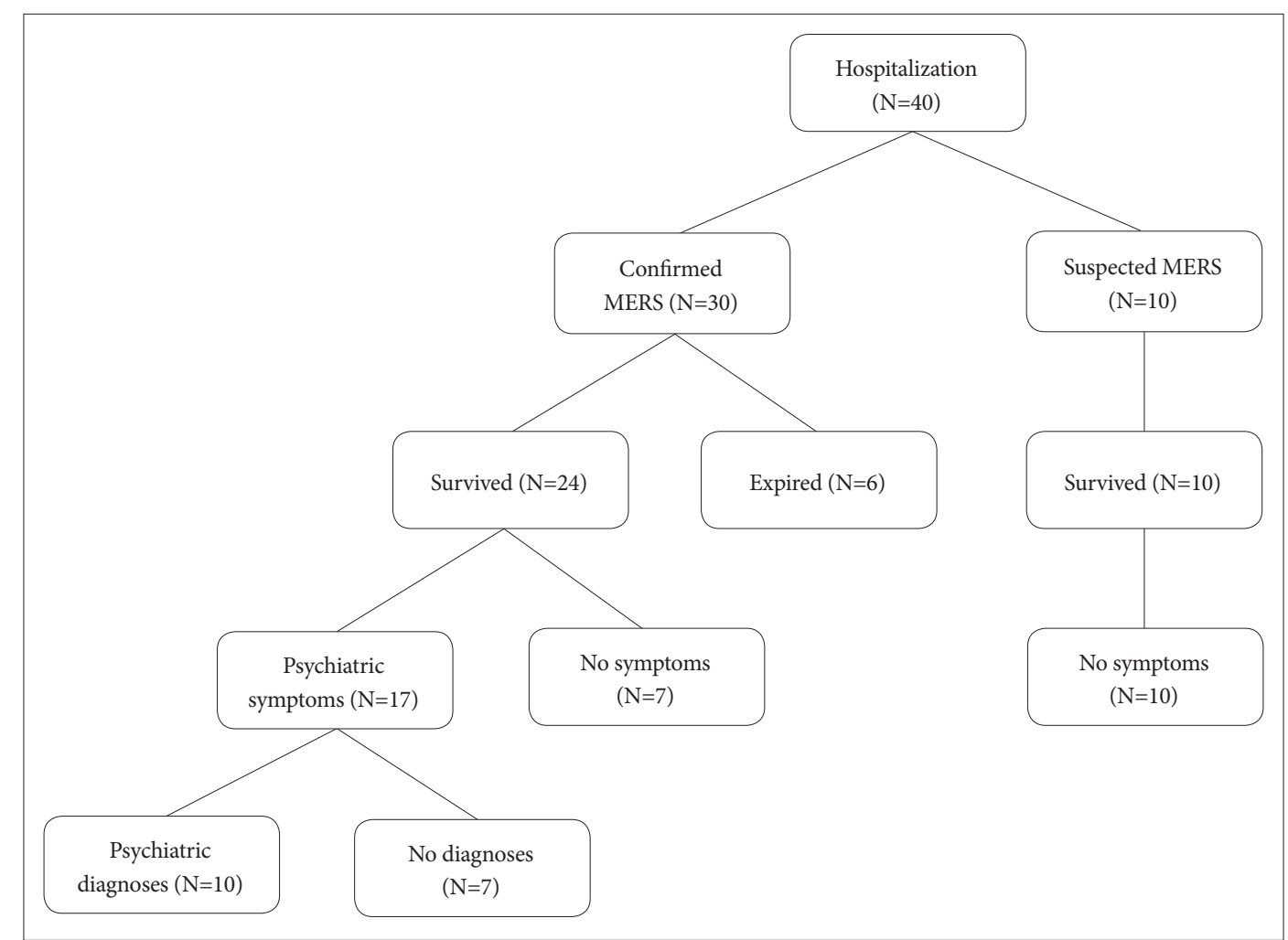

Figure 1. Psychiatric complications in patients with MERS during the acute treatment phase. MERS: Middle East respiratory syndrome. 
with dementia deteriorated further after the MERS infection and isolation. Moreover, a patient who lost her father to MERS experienced bereavement during isolation.

\section{Description of difficult cases}

Most of the patients had no knowledge of MERS. Some asked whether they would carry the virus and be infectious for life. Some patients feared impending death, and two patients previously diagnosed with panic disorder experienced panic attacks although the panic disorder had been in remission before contracting MERS.

Several patients reported distress from not knowing the effects of MERS and feelings of isolation, as well as concerns about their family and workplace colleagues. Several patients reported feelings of guilt and fear of spreading MERS. One patient felt that he was being punished for something as an explanation for why he had been infected.

One patient had been infected by her father, whom she had nursed in the hospital. Her father had died of MERS-related complications. She received this news while in isolation herself. She was unable to attend his funeral, as all deceased patients were cremated shortly after their deaths and formal funerals were impossible. She felt depressed and sad.

An elderly patient did not understand why she was restricted to a room after she had recovered consciousness. She cried every day, begged the nurses to let her go home, and occasionally expressed anger and made suicidal threats.

A blind patient who had lost contact with his healthcare aide was so mistrustful of the medical staff that he initially refused admission.

\section{Psychological assessment}

Psychological data were not obtained from 7 of the 34 survivors due to intubation, delirium, cognitive dysfunction, or noncompliance. Thus, 27 (79.4\%) survivors completed the psychological assessment and were included in the analysis. Of those, 18 (66.7\%) were confirmed and $9(33.3 \%)$ were suspected MERS cases, 10 (37.0\%) patients were male and 17 (63.0\%) were female, and the mean age was $41.15 \pm 18.64$ (range, $21-83$ ) years. In total, 15 (55.6\%) patients reported previous medical conditions and 2 (7.4\%) reported previous psychiatric disorders (Table 1).

According to the PHQ-9 criteria, 11 (40.7\%) participants were depressed (PHQ-9 $\geq 5$ ) and 16 (59.3\%) were not depressed. Patients with mild-to-severe depression had significantly higher mean scores on the KNHANES-short form $(\mathrm{t}=4.169, \mathrm{p}<$ 0.001), IES-R ( $\mathrm{t}=2.262, \mathrm{p}=0.017)$, and PTD $(\mathrm{t}=2.922, \mathrm{p}=0.007)$ than those without depression. However, the mean PTN and PTSS scores were not significantly different between groups (Table 2).

Table 1. General characteristics of patients who completed the psychological assessment

\begin{tabular}{|c|c|c|c|c|}
\hline Variables & $\begin{array}{l}\text { Total } \\
(\mathrm{N}=27)\end{array}$ & $\begin{array}{c}\text { Confirmed MERS } \\
\text { patients }(\mathrm{N}=18)\end{array}$ & $\begin{array}{c}\text { Suspected MERS } \\
\text { Patients }(\mathrm{N}=9)\end{array}$ & Statistics ${ }^{\dagger}$ \\
\hline Gender, N (\%) & & & & $\chi^{2}=1.271, p=0.406$ \\
\hline Male & $10(37.0)$ & $8(44.4)$ & $2(22.2)$ & \\
\hline Female & $17(63.0)$ & $10(55.6)$ & $7(77.8)$ & \\
\hline Age (year) (mean $\pm S D)$ & $41.15 \pm 18.64$ (range $21-83)$ & $46.33 \pm 18.85$ & $30.78 \pm 13.89$ & $\mathrm{t}=-2.187, \mathrm{df}=25, \mathrm{p}=0.038$ \\
\hline Marital status, $\mathrm{N}(\%)$ & & & & $\chi^{2}=4.120, p=0.127$ \\
\hline Single & $11(40.7)$ & $5(27.8)$ & $6(66.7)$ & \\
\hline Divorced/separated & $2(7.4)$ & $2(11.1)$ & $0(0)$ & \\
\hline Married & $14(51.9)$ & $11(61.1)$ & $3(33.3)$ & \\
\hline Education, N (\%) & & & & $\chi^{2}=0.750, p=0.667$ \\
\hline High school or lower & $9(33.3)$ & $7(38.9)$ & $2(22.2)$ & \\
\hline More than high school & $18(66.7)$ & $11(61.1)$ & $7(77.8)$ & \\
\hline Health care provider*, N (\%) & & & & $\chi^{2}=4.747, p=0.046$ \\
\hline Yes & $13(48.1)$ & $6(33.3)$ & $7(77.8)$ & \\
\hline No & $14(51.9)$ & $12(66.7)$ & $2(22.2)$ & \\
\hline Previous medical disease, N (\%) & & & & $\chi^{2}=0.000, p=1.000$ \\
\hline Yes & $15(55.6)$ & $10(55.6)$ & $5(55.6)$ & \\
\hline No & $12(44.4)$ & $8(44.4)$ & $4(44.4)$ & \\
\hline Previous psychiatric disorders, N (\%) & & & & $\chi^{2}=1.080, p=0.538$ \\
\hline Yes & $2(7.4)$ & $2(11.1)$ & $0(0)$ & \\
\hline No & $25(92.6)$ & $16(88.9)$ & $9(100)$ & \\
\hline
\end{tabular}

*a kind of health care provider included medical doctor, nurse, and nurse assistant among job, ${ }^{\dagger}$ variables were compared between Confirmed MERS patients and Suspected MERS Patients. MERS: Middle East Respiratory Syndrome 
Table 2. Psychological variables according to depression status $(\mathrm{N}=27)$

\begin{tabular}{lccc}
\hline \multicolumn{1}{c}{ Variables } & $\begin{array}{c}\text { No. depression } \\
\text { PHQ- } 9 \leq 4(\mathrm{~N}=16)\end{array}$ & $\begin{array}{c}\text { Depression* } \\
\text { PHQ-9 } \geq 5(\mathrm{~N}=11)\end{array}$ & Statistics \\
\hline KNHANES-Short form (mean \pm SD) & $11.06 \pm 2.38$ & $20.64 \pm 8.80$ & $\mathrm{t}=4.169, \mathrm{df}=25, \mathrm{p}<0.001$ \\
IES-R & $5.31 \pm 8.36$ & $18.09 \pm 17.34$ & $\mathrm{t}=2.262, \mathrm{df}=25, \mathrm{p}=0.017$ \\
PTD-PTNB-PTSS (mean \pm SD) & & & \\
PTD & $3.63 \pm 1.09$ & $7.18 \pm 4.73$ & $\mathrm{t}=2.922, \mathrm{df}=25, \mathrm{p}=0.007$ \\
PTNB & $12.50 \pm 3.08$ & $14.73 \pm 5.87$ & $\mathrm{t}=1.289, \mathrm{df}=25, \mathrm{p}=0.209$ \\
PTSS & $5.19 \pm 0.54$ & $6.73 \pm 3.58$ & $\mathrm{t}=1.707, \mathrm{df}=25, \mathrm{p}=0.100$ \\
\hline
\end{tabular}

*patients with depression (PHQ-9 $\geq 5)(\mathrm{N}=11)$ included 8 subjects (29.6\%) with mild depression (PHQ-9, 5-9), 2 (7.4\%) with moderate depression (PHQ-9, 10-14), and 1 (3.7\%) with severe depression (PHQ-9, 20-27). PHQ-9: Patient Health Questionnaire-9, IES-R: the Impact of Event Scale-Revised, PTD-PTNB-PTSS: Peri-traumatic Dissociation-Post-traumatic Negative Beliefs-Post-traumatic Social Support, KNHANES: National Health and Nutrition Examination Survey

Table 3. Correlations between PHQ-9 and the other psychological test scores

\begin{tabular}{|c|c|c|c|c|c|c|}
\hline & \multirow{2}{*}{ PHQ-9 } & \multirow{2}{*}{ KNHANES-short form } & \multirow{2}{*}{ IES-R } & \multicolumn{3}{|c|}{ PTD-PTNB-PTSS } \\
\hline & & & & PTD & PTNB & PTSS \\
\hline PHQ-9 & 1 & & & & & \\
\hline KNHANES-short form & $0.814^{* *}$ & 1 & & & & \\
\hline IES-R & $0.679^{* *}$ & $0.626^{* *}$ & 1 & & & \\
\hline \multicolumn{7}{|l|}{ PTD-PTNB-PTSS } \\
\hline PTD & $0.627^{* *}$ & $0.770^{* *}$ & $0.630^{* *}$ & 1 & & \\
\hline PTNB & $0.454^{*}$ & $0.564^{* *}$ & $0.751^{* *}$ & $0.571^{* *}$ & 1 & \\
\hline PTSS & 0.363 & $0.594^{* *}$ & $0.786^{* *}$ & $0.627^{* *}$ & $0.772^{* *}$ & 1 \\
\hline
\end{tabular}

${ }^{*} \mathrm{p}<0.05,{ }^{* *} \mathrm{p}<0.001$. PHQ-9: Patient Health Questionnaire-9, IES-R: the Impact of Event Scale-Revised, PTD-PTNB-PTSS: Peri-traumatic Dissociation-Post-traumatic Negative Beliefs-Post-traumatic Social Support, KNHANES: National Health and Nutrition Examination Survey

Table 4. Analysis of the relationship between PHQ-9 scores and the IES-R and KNHANES-short form scores

\begin{tabular}{|c|c|c|c|c|c|}
\hline & $\mathrm{B}$ & Standard error & $\beta$ & $\mathrm{t}$ & $\mathrm{p}$ \\
\hline (Constant) & 0.543 & 2.211 & & 0.246 & 0.808 \\
\hline KNHANES-short form & 0.485 & 0.118 & 0.730 & 4.120 & $<0.001$ \\
\hline IES-R & 0.167 & 0.063 & 0.473 & 2.642 & 0.015 \\
\hline PTD & -0.113 & 0.251 & -0.080 & -0.451 & 0.656 \\
\hline PTNB & -0.297 & 0.186 & -0.267 & -1.597 & 0.125 \\
\hline
\end{tabular}

PHQ-9: Patient Health Questionnaire-9, IES-R: the Impact of Event Scale-Revised, PTD-PTNB-PTSS: Peri-traumatic Dissociation-Posttraumatic Negative Beliefs-Post-traumatic Social Support, KNHANES: National Health and Nutrition Examination Survey

The PHQ-9 scores were significantly positively correlated with the KNHANES-short form $(r=0.814, \mathrm{p}<0.001)$, IES-R $(\mathrm{r}=0.679, \mathrm{p}<0.001)$, PTD $(\mathrm{r}=0.627, \mathrm{p}<0.001)$, and PTNB $(\mathrm{r}=$ $0.454, \mathrm{p}=0.017$ ) scores. Conversely, the PHQ-9 scores were not significantly correlated with the PTSS scores (Table 3).

We performed a multiple linear regression analysis to assess the impact of the psychological factors measured by the IESR, PTD, PTNB, and KNHANES-short form on depression severity in suspected and confirmed MERS patients. The IESR, PTD, PTNB, and KNHANES-short form scores yielded an adjusted $\mathrm{R}^{2}$ value of $0.693(\mathrm{~F}=12.747, \mathrm{p}<0.001)$. The analysis revealed that only the KNHANES-short form and IES-R scores were significantly positively correlated with PHQ-9 scores (Table 4).

\section{DISCUSSION}

We found that about 70\% of the confirmed MERS patients exhibited psychiatric symptoms and about $40 \%$ received psychiatric diagnoses and were prescribed medication during hospital quarantine, whereas none of the suspected MERS patients exhibited psychiatric symptoms. Furthermore, we found that the level of daily distress over the past month and posttraumatic stress symptoms may be risk factors for depression 
among suspected or confirmed MERS patients during the hospital stay.

A previous study found that seven of ten SARS patients with psychiatric complications were deemed to have mild psychiatric problems such as anger, anxiety, suicidal ideas, and depressive reaction, whereas the remaining three patients had more severe psychiatric disorders with hallucinatory and manic features. Similarly, we found that 14 of 24 MERS patients had mild psychiatric symptoms such as insomnia, depressed mood, and tension and that three had severe psychiatric disorders with hallucinations and psychotic features.

The recent outbreaks of SARS in Asia and Canada provide the most recent data on emerging infectious diseases (EIDs). Psychiatric disorders and chronic fatigue were prevalent among SARS survivors 3 years after contracting the disease and were associated with various functional impairments. ${ }^{7}$ However, to our knowledge, our study is the first to use selfreported measures to assess risk factors for the development of psychiatric symptoms during the acute treatment period. About 35\% of SARS survivors reported moderate-to-severe or severe anxiety and/or depressive symptoms 1 month after recovery. ${ }^{8}$ Moreover, SARS survivors had elevated levels of psychological distress 1 year after the outbreak, and 64\% scored above the General Health Questionnaire cut-off for psychiatric morbidity. ${ }^{9}$ The current prevalence for any psychiatric disorder 30 months post-SARS is $33.3 \% .^{10}$

A biopsychosocial model may explain the development of psychiatric symptoms in patients with MERS. Relevant biological factors may be weight loss of $10 \mathrm{~kg}$ to $20 \mathrm{~kg}$, ${ }^{11}$ antiviral agent side effects, ${ }^{12}$ pre-existing disabilities (e.g., blindness or hearing impairment), or a history of psychiatric disorders. Relevant psychological factors include tension, fear, anger, mistrust, and depressed mood due to MERS and subsequent isolation during quarantine. ${ }^{2}$ Additionally, we found that the level of daily distress over the past month and post-traumatic stress symptoms may be risk factors for depression during acute hospital quarantine. Social factors that may have led to unhappiness during treatment include financial losses attributable to isolation and separation from young children or parents at home.

Given the nature and characteristics of MERS, we established a patient-centered consultation program to monitor the safety issues that arose during the period of total isolation and offered mental health services to all MERS inpatients, regardless of whether they were diagnosed with psychiatric disorders. Our guiding principle was centered on the need for early detection of psychiatric symptoms including those related to difficulty in accessing MERS wards.

Our study has potential limitations. We used a retrospective chart review design performed in a single center with a small study population. Furthermore, our study was not designed to compare depressed and non-depressed patients. Additionally, the small sample size may have affected the prevalence rate of psychiatric symptoms and treatment.

Despite these limitations, our findings indicate that the acute treatment of MERS-CoV infections in quarantine had a significant impact on the patients' mental health. Furthermore, assessment of the risk factors for depression may identify vulnerable patients who require psychiatric care and attention during hospital quarantine. Our findings suggest the need to increase mental health services during the acute treatment phase of EIDs and to conduct follow-up studies in survivors.

\section{Acknowledgements}

This research was supported by a grant of the Korea Health Technology R\&D Project through the Korea Health Industry Development Institute (KHIDI), funded by the Ministry of Health \& Welfare, Republic of Korea (HI15C3227).

\section{REFERENCES}

1. Cho SY, Kang JM, Ha YE, Park GE, Lee JY, Ko JH, et al. MERS-CoV outbreak following a single patient exposure in an emergency room in South Korea: an epidemiological outbreak study. Lancet 2016;388:9941001.

2. Cheng SKW, Tsang JSK, Ku KH, Wong CW, Ng YK. Psychiatric complications in patients with severe acute respiratory syndrome (SARS) during the acute treatment phase: a series of 10 cases. Br J Psychiatry 2004;184:359-360.

3. Kroenke K, Spitzer RL. The PHQ-9: a new depression diagnostic and severity measure. Psychiatr Ann 2002;32:509-515.

4. Lim HK, Woo JM, Kim TS, Kim TH, Choi KS, Chung SK, et al. Disaster Psychiatry Committee in Korean Academy of Anxiety Disorders. Reliability and validity of the Korean version of the Impact of Event Scale-Revised. Compr Psychiatry 2009;50:385-390.

5. Lee ES, Shin HC, Yang YJ, Cho JJ, Ahn KY, Kim SH. Development of the Stress Questionnaire for KNHANES: Report of Scientific Study Service. Seoul: Korea Centers for Disease Control and Prevention; 2010.

6. Joo H, Ahn H. Development and posttrauma risk checklist. Korean J Psychol Gen 2008;27:235-225.

7. Wing YK, Leung CM. Mental health impact of severe acute respiratory syndrome: a prospective study. Hong Kong Med J 2012;18(Suppl 3):2427.

8. Cheng SK, Wong CW, Tsang J, Wong KC. Psychological distress and negative appraisals in survivors of Severe Acute Respiratory Syndromes (SARS). Psychol Med 2003;34:1187-1195.

9. Lee AM, Wong JGWS, McAlonan GM, Cheung V, Cheung C, Sham PC, et al. Stress and psychological distress among SARS survivors 1 year after the outbreak. Can J Psychiatry 2007;52:233-240.

10. Mak IWC, Chu CM, Pan PC, Yiu MGC, Chan VL. Long-term psychiatric morbidities among SARS survivors. Gen Hosp Psychiatry 2009;31: 318-326.

11. Stanga Z, Field J, Iff S, Stucki A, Lobo DN, Allison SP. The effect of nutritional management on the mood of malnourished patients. Clin Nutr 2007;26:379-382.

12. Este JA, Cihlar T. Current status and challenges of antiretroviral research and therapy. Antivir Res 2010;85:25-33. 